\title{
PRODUCTION PRIMAIRE, PRODUCTION UTILE : MÉTHODES D'ÉVALUATION, INDICES DE PRODUCTIVITÉ
}

\author{
N. DECOURT \\ Station de Sylviculture et de Production, \\ Centre national de Recherches forestières, I. N. R. A., \\ Champenoux 54370 Einville
}

\begin{abstract}
RÉSUMÉ
Les termes employés sont définis dans une première partie. S'appuyant sur des travaux récents, le problème des méthodes d'évaluation est abordé en signalant chaque fois l'analogie entre les méthodes concernant le volume et celles concernant la biomasse. Enfin, la notion d'indice de productivité est reliée à celle de loi de croissance. Une hypothèse est proposée conduisant à considérer l'index foliaire comme un bon indice de la productivité primaire.
\end{abstract}

Le sujet à traiter étant très vaste, je me bornerai à un rappel des définitions des termes employés, puis à des notions assez générales sur les méthodes d'évaluation, en me réservant ainsi la possibilité d'insister un peu plus sur le problème des indices.

\section{I. - DÉFINITIONS}

La Biomasse sera la masse de l'organisme vivant considéré ou celle des individus formant une population ou celle des populations formant une communauté (Duvigneaud, 1967).

Dans l'Ecosystème, on distingue traditionnellement plusieurs niveaux d'activité (niveaux de production, de consommation, de décomposition...).

Au niveau de production, fonctionnent les organismes utilisant l'énergie lumineuse pour construire la matière vivante : essentiellement les végétaux chlorophylliens et dans un écosystème forestier essentiellement les arbres. Cette matière vivante ne se réduit pas aux matières organiques produites par la synthèse chlorophyllienne et par les réactions qui y 
font suite. Elle inclut des éléments minéraux indispensables à la vie des organismes producteurs. II n'est donc pas logique comme certains écologistes le proposent, de séparer artificiellement ces éléments minéraux (minéralomasse) de la biomasse.

Plus généralement, il nous semble légitime d'inclure dans la biomasse toute la matière organique ou non, vivante ou non, organisée de façon permanente pour constituer les êtres vivants (le bois de cœur doit donc y être inclus).

La vitesse de production de la biomasse est appelée productivité. L'accroissement de la biomasse par unité de temps sera la productivité nette, qu'on définit ainsi comme la différence entre la matière assimilée et les pertes diverses (pour les végétaux essentiellement la respiration).

La productivité des producteurs est la productivité primaire. Elle sera donc égale à l'accroissement de la biomasse des végétaux chlorophylliens.

Dans un écosystème forestier, cette biomasse végétale est formée essentiellement par les arbres, qui constituent même souvent des peuplements d'une même espèce (que l'écologiste appellera population et le forestier peuplement pur) ou parfois par le mélange de quelques espèces (on parlera alors de cominunauté ou de peuplement mélangé). Si les arbres sont d'âges égaux ou voisins, on parlera de peuplements équiennes. Les peuplements presque purs et peu mélangés sont fréquents, non seulement dans les forêts aménagées par l'homme, mais également dans les forêts "naturelles ", tout au moins en zones tempérée et froide.

La production utile est évidemment une notion beaucoup moins bien définie que celle de biomasse ou de productivité, puisqu'elle dépend de l'usage qu'on fait d'une partie variable de la productivité primaire, partie qui est prélevée par une récolte.

En général, c'est une fraction du volume ligneux qui est prélevée par le forestier, qui traditionnellement parle plus volontiers en terme de volume qu'en terme de masse, encore qu'avec les usages modernes du bois, pour la fabrication du papier et de la cellulose, l'emploi de la masse devient de plus en plus fréquent.

Le pourcentage de la productivité primaire récolté de façon utile par le forestier est donc extrêmement variable. Le forestier allemand WECK, J. (1960) estime qu'il faut considérer d'abord la récolte forestière potentielle en soustrayant à la productivité totale les feuilles, les écorces, une partie des branches, les racines et les souches.

Ce potentiel serait de loordre dans nos régions de $32 \%$ et dans la zone des pluies tropicales de $14 \%$ de l'assimilation totale. Mais, si on tient compte de l'action des divers parasites (champignons, insectes) et des pertes lors de la récolte, on ne récolterait en fait que $75 \%$ de ce potentiel dans nos régions et $35 \%$ dans la zone des pluies tropicales.

Si oi considère la production utile de boos dérinie de fason encore plus restrictive par des critères de qualité, comme la prowuction en hois d'ixuvre par exemcle, ces pourcentages sont évidemment encore plus faibles. I. vuhleci 1 illustre ces considérations en donnant approximativement la contribution des grands ty acs ci forâts à la production de matière sèche, à la récolte en bois et à la récolte en bovis d'ưuvre.

La notion de volu me bois-fort, volume mesuré sur écorce, des bois de circonférence supérieura à $22 \mathrm{~cm}$ est une approximation commosss, et acceptéa par l'Union Internationale des Stations de Recherches forestières, four exprimer le volume utile de la masse ligneuse, sur pied ou récoltée, dans les peuplements forestiers.

On considérera done la biomusse végýtule et son aceroissement, la productivité primuire 
TABLEAU 1

Production potentielle et production utile (en \% de la masse)

Classes de formation
Forêts tropicales

Forêts de Lauracées et arbres à feuilles dures

Forêts à feuilles caduques avec inclusions de conifères de montagne ............

Forêts boréales de conifères.

\section{$\%$ de la surface des forêts du globe}

$\%$ de la
production
de matière
sèche

61

7

19,5

12,5

$$
\begin{aligned}
& \% \text { de } \\
& \text { la récolte } \\
& \text { totale en }
\end{aligned}
$$
bois

22

11

39

28
Bois

d'œuvre

d'après WECK J. (1960).

(essentiellement celle des arbres, dans les écosystèmes forestiers), et d'autre part le volume de bois et son accroissement. Il s'agit généralement d'accroissement annuel, et ces quantités sont en général rapportées à l'unité de surface.

Il est important de bien souligner ici les liens qui unissent ces variables, biomasse ou volume, à leurs accroissements. Les accroissements sont les dérivés des variables considérées. Au cours du temps leur valeurs se modifient (sauf dans les peuplements parfaitement en équilibre). On sait que dans les peuplements équiennes, au cours de la vie du peuplement, les accroissements annuels croissent, puis passent par un maximum pour décroître ensuite dans les vieux peuplements.

L'accroissement moyen par année d'âge, depuis l'origine du peuplement, suit une évolution analogue, passant par un maximum au moment précis où il devient égal à l'accroissement (voir figure 1). Pour distinguer clairement ces deux notions, on parle d'accroissement courant annuel et d'accroissement moyen depuis l'origine.

$$
\begin{aligned}
& A c c^{\dagger} \\
& \left(m^{3} / h a / a n\right)
\end{aligned}
$$

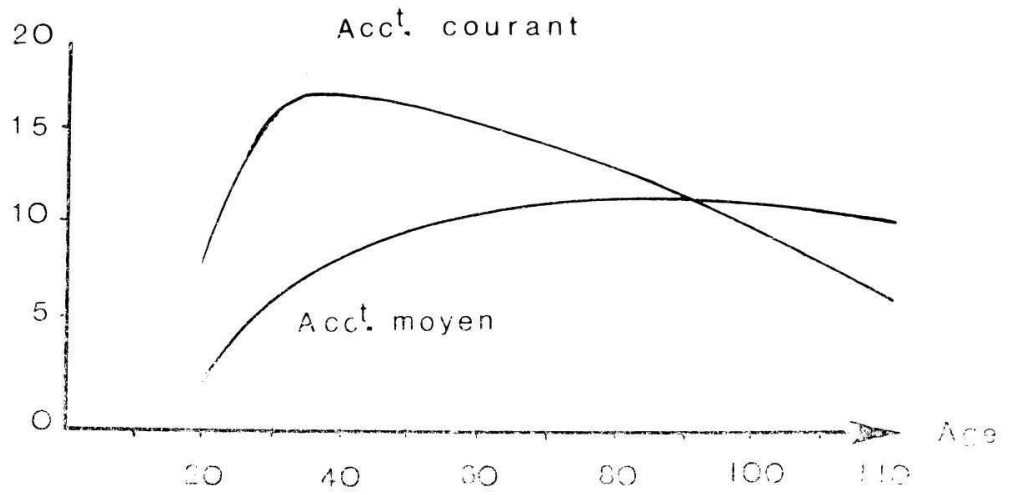

FIG. 1. - Accroissement moyen et accroissement courant en fonction de l'âge

(D'après la table de production de Wiedemann - classe I-Épicéa Commun) 
La relation évoquée ci-dessus est une relation mathématique, qui n'est pas liée à la biologie des forêts, mais à la définition des variables. La perdre de vue et confondre accroissement courant et accroissement moyen conduit bien entendu à des résultats aberrants.

\section{II. - MÉTHODES D’ÉVALUATION}

L'évaluation des volumes et plus encore des biomasses, puis de leurs accroissements, n'est pas chose facile. La biomasse des différentes parties de l'arbre, puis du peuplement, est plus ou moins difficile à mesurer. Le problème des racines, par exemple, est particulièrement ardu.

On peut, bien sûr, faire des mesures directes, à condition de disposer des moyens nécessaires. Ces mesures sont très coûteuses et détruisent l'objet mesuré. On ne peut les répéter un grand nombre de fois.

Les mesures indirectes, plus intéressantes, posent, qu'il s'agisse de volume ou de biomasse, les mêmes types de questions : il s'agit de problème de tarif et de problème d'échantillonnage, au niveau des arbres.

\section{1. - Évaluation des volumes ou des biomasses des arbres. Tarifs}

On désire évaluer un paramètre descriptif d'un arbre comme son volume, sa biomasse, la surface de ses feuilles, etc... Ce paramètre $\mathrm{Y}$ est difficile à mesurer directement. On cherche alors à exprimer $\mathrm{Y}$ en fonction d'un certain nombre de paramètre $\mathrm{X}_{1}, \mathrm{X}_{2} \ldots \mathrm{X}_{n}$ qui eux sont plus faciles à mesurer (circonférence à hauteur d'homme, hauteur totale, etc.).

On aura :

$$
\mathrm{Y}=f\left(\mathrm{~A}_{1}, \mathrm{~A}_{2} \ldots \mathrm{A}_{p}, \mathrm{X}_{1}, \mathrm{X}_{2} \ldots \mathrm{X}_{n}\right) \text {. }
$$

$\mathrm{Y}$ est une fonction, plus ou moins compliquée des $\mathrm{X}_{1}, \mathrm{X}_{2} \ldots$ mesurables sur les arbres. Les paramètres $\mathrm{X}_{1}, \mathrm{X}_{2} \ldots \mathrm{X}_{n}$ sont les entrées du tarif. $\mathrm{A}_{1}, \mathrm{~A}_{2} \ldots \mathrm{A}_{p}$ sont généralement des constantes, au moins pour un groupe d'arbres déterminés (arbres d'une même espèce, ou d'un même peuplement par ex.). $A_{1}, A_{2} \ldots A_{p}$ peuvent donc prendre des valeurs différentes pour ces différents groupes d'arbres. On les considérera comme des paramètres du tarif.

Enfin, la forme même de la fonction $f$ peut n'être valable que pour certains groupes d'arbres. Il y aura alors des tarifs différents pour chacun de ces groupes.

Exemple : $v=a+b \mathrm{C}^{2}$ est un tarif utilisé dans les peuplements équiennes et purs. Il s'agit d'un tarif à une entrée $C$, qui est la circonférence à $1,30 \mathrm{~m}$ et qui donne le volume ligneux d'un arbre. $a$ et $b$ sont des constantes qui dépendent du peuplement (essence, station, densité, etc.).

Le problème des tarifs est donc de toute évidence étroitement relié au problème très général des relations d'allométrie chez les êtres vivants. Il s'agit finalement de trouver quelles relations invariantes existent entre les différents paramètres descriptifs des arbres.

Pour les volumes ligneux et pour des raisons évidentes, un nombre considérables d'études existe sur ce sujet (PARDe 1961, Bouchon 1972). Pour la biomasse des différents composants (tiges, branches, feuilles, racines) des arbres, il est remarquable de constater dans la bibliographie (RIEDACKER 1968, 
Young 1971) que la démarche suivie a été exactement la même. On a notamment recherché, avec un certain succès, des relations du type :

$$
\begin{aligned}
& \log \mathrm{P}=a+b \log \mathrm{D}_{1}{ }^{30} \\
& \log \mathrm{P}=a+b \log \mathrm{D}_{1,30}+c \log \mathrm{H}, \\
& \log \mathrm{P}=a+b \log \mathrm{D}_{b} \text { (pour la masse foliaire), } \\
& \mathrm{P}=\text { poids du composant, } \\
& \mathrm{D}_{1,30}=\text { diamètre de l'arbre à 1,30, } \\
& \mathrm{H}=\text { hauteur totale, } \\
& \mathrm{D}_{b}=\text { diamètre de l'arbre à la base du houppier, }
\end{aligned}
$$

$a, b, c=$ constantes caractérisant l'espèce, le peuplement, etc. certains auteurs estimant qu'elles sont caractéristiques de l'espèce, d'autres qu'il faut tenir compte également d'autres facteurs.

On retiendra donc que pour les composants de la biomasse, comme du reste pour la biomasse totale et comme, plus traditionnellement, pour le volume, on commence à connaître des relations d'allométrie du type : $(\mathrm{V}$. ou $\mathrm{P})=f\left(\mathrm{C}_{1,30}, \mathrm{H}\right)$ ou $(\mathrm{V}$ ou $\mathrm{P})=f\left(\mathrm{C}_{130}\right.$, $\left.\mathrm{H}, \mathrm{C}_{i}\right), \mathrm{C}_{i}$ étant une circonférence mesurée parfois à un autre niveau.

\section{2. - Problème du passage au peuplement}

Le passage au peuplement se fait par l'intermédiaire d'une autre unité d'échantillonnage, la placette qui est choisie comme représentative du peuplement, ce qui pose un certain nombre de problèmes qui ne seront pas abordés ici.

Là encore, le fait important est constitué par la convergence des méthodes et de façon plus remarquable sans doute par la convergence des résultats en ce qui concerne l'évaluation classique des volumes et celle de la biomasse ou de ses constituants.

Considérons par exemple le tarif à une entrée signalé ci-dessus :

$$
v=a+b \mathrm{C}^{2} \text {, }
$$

valable pour les arbres d'un peuplement pur et équienne donné, comportant $\mathrm{N}$ tiges. Le volume de ce peuplement, soit V, sera calculé comme la somme des volumes individuels des arbres :

$$
\mathrm{V}=\Sigma v=\mathrm{N} a+b \Sigma \mathrm{C}^{2}
$$

$\mathrm{Ou}$, si on considère la surface terrière $\mathrm{G}$ du peuplement

$$
\mathrm{V}=\mathrm{N} a+4 \pi b . \mathrm{G}
$$

Une telle formule, bien établie, donne un moyen simple de calculer V. De plus, elle souligne que l'arbre de surface terrière moyenne est aussi l'arbre de volume moyen du peuplement.

Pour évaluer le volume d'un peuplement, on pourra donc se contenter de mesurer un certain nombre de tiges échantillon pour calculer un tarif valable pour le peuplement (méthode par régression). On n'aura plus ensuite qu'à mesurer les seules entrées du tarif sur les arbres du peuplement. Mieux, certaines formules suggèrent que pour le volume, la mesure de quelques arbres moyens bien choisis pourrait suffire (méthode de l'arbre moyen).

Dans le cas de l'estimation des biomasses, après une période de tâtonnement, on s'est très vite orienté vers ces deux types de méthodes. Les travaux les plus récents montrent que 
la méthode par régression est très suffisante (MADGWICK, 1971). Dans le cas de peuplement équienne et pur, on peut, comme pour le volume ligneux, utiliser comme arbre moyen l'arbre de surface terrière moyenne (CROW, 1971).

\section{3. - Les accroissements}

Le problème se complique quand il s'agit d'estimer des accroissements courants annuels en volume, ou d'évaluer la productivité primaire nette.

Les méthodes d'estimations directes par comparaison d'inventaire pratiquées parfois pour les accroissements en volume, notamment dans les placettes d'expériences permanentes sont impraticables dans la pratique pour la biomasse. On s'oriente là encore, pour le volume comme pour la biomasse, soit sur la mesure directe des accroissements ligneux annuels (cernes annuels) dans le cas du bois, soit et parfois simultanément, sur la dérivation des tarifs. Dans le cas de la masse foliaire P par exemple, si on possède un bon tarif du type :

$\mathrm{P}=f(\mathrm{D}) \quad$ (D étant un diamètre de la tige moyenne par exemple)

on aura :

$$
\frac{d \mathrm{P}}{d t}=f_{1}, \frac{d \mathrm{D}}{d t},
$$

$d \mathrm{D}$ $d t$

pouvant s'apprécier à partir de la mesure des cernes annuels au niveau du diamètre considéré. Cette méthode suppose qu'on néglige, ou qu'on évalue par ailleurs la mortalité, ou la perte de substance supportée par l'objet mesuré pendant l'intervalle de temps $d t$.

Lorsqu'il s'agira d'évaluer la production totale (en volume ou en biomasse) sur une longue période de temps, ou même depuis l'origine, le problème se compliquera encore, puisque cette production $\Sigma \mathrm{V}$, comporte deux termes :

$\Sigma \mathrm{V}=\mathrm{V}+\Sigma v$

$\mathrm{V}=$ Volume (ou Biomasse) du peuplement actuel,

$\Sigma v=$ somme des volumes ou des biomasses disparus par mortalité naturelle ou provoquée (éclaircie) durant la période considérée.

L'évaluation rigoureuse de $\Sigma \mathrm{V}$ ne peut donc provenir que de la mesure soigneuse et suivie de placettes permanentes pendant toute leur vie. Le C.N.R.F. possède ainsi des placettes mesurées régulièrement depuis parfois plus de 100 ans et dont on connaît la production totale en volume depuis l'origine. Ces données n'existent sans doute jamais pour la biomasse.

En l'absence de placettes permanentes, le problème consiste à évaluer la production totale à partir de la connaissance des accroissements courants annuels. On sait que ce dernier est une fonction de l'âge des peuplements. Il faudra donc rechercher quelle est cette fonction, ce qui implique un nombre considérable de mesures dans un ensemble de peuplements d'âge varié, en faisant l'hypothèse souvent risquée que ces peuplements suivent la même loi de croissance.

C'est la méthode pratiquée pour des peuplements équiennes et purs (ou sensiblement équiennes et presque purs) lorsqu'on construit des tables de production en volume, 
à partir de placettes temporaires (Vuokila 1967, Decourt 1971). La manière de poser le problème serait la même pour la biomasse.

Dans le cas de peuplements réputés en équilibre, c'est-à-dire identiques à eux-mêmes dans le temps, et en moyenne, sur une surface suffisamment faible, le problème serait en théorie facilité, puisque l'accroissement en biomasse devrait être constamment nul. Le problème se pose de savoir si de tels peuplements existent réellement.

On peut déjà souligner ici que l'étude des stations et l'utilisation d'indices de station est sans doute un des moyens de grouper les peuplements en classes supposées caractérisée par une même loi de croissance.

\section{4. - Conclusion - Deux remarques}

La mesure des masses comme celle des volumes, et l'évaluation de leurs accroissements font appel à la notion de tarif qui n'est qu'une application particulière de l'existence très générale de relations d'allométrie entre les paramètres descriptifs des êtres vivants (HUXLEY, J. 1932).

Ces relations expriment sans doute, comme le souligne Rosen R. (1967) des équilibres fonctionnels. Jusqu'à présent, les études dans ce domaine ne visaient qu'à rechercher, généralement par régression, des formules permettant de faciliter un travail de prédiction dans des cas plus ou moins particuliers.

De nouveaux progrès ne seront possibles que par l'étude systématique de l'architecture des arbres et des peuplements, c'est-à-dire par des études de morphométrie, conduisant à l'établissement de forme générale (et théorique) pour les fonctions tarifs, dont il suffira de connaître ensuite les paramètres et les entrées (voir II, 1).

Le fait que les entrées semblent les mêmes pour l'estimation des volumes et des composants de la biomasse, et notamment la valeur représentative de l'arbre de surface terrière moyenne pour certains types de peuplements, permettra, lorsque les tarifs seront mieux connus, de faire bénéficier les études sur la biomasse et la productivité primaire de l'énorme masse de données accumulées pour l'étude des volumes dans les différentes stations de recherches forestières.

\section{III. - INDICES DE PRODUCTIVITÉ}

Il se produit pour l'accroissement courant en volume, comme du reste pour la productivité primaire, un peı le même phénomène que pour la mesure des volumes ou des biomasses et le calcul de leurs accroissements, mais à un niveau supérieur. Pour plus de facilité, nous nous limiterons d'abord à l'appréciation de la productivité en volume.

\section{1. - Position du problème}

Comme nous l'avons évoqué ci-dessus, la productivité et plus encore la production totale sont difficiles à atteindre directement (placettes permanentes), ou à évaluer par le calcul (placettes temporaires). Comme pour les tarifs, on a donc cherché des paramètres simples caractérisant la productivité (et donc la production totale) d'une station pour une espèce donnée, ou pour plusieurs espèces. 
Cette idée repose sur l'hypothèse que dans une même station, un matériel végétal donné suit en moyenne une même loi de croissance en volume.

La chose paraît évidente. Seule l'accumulation de nombreuses données, notamment dans des placettes permanentes a cependant permis de l'établir. En effet, la productivité d'un même matériel végétal dépend de nombreux facteurs de production : climatiques, pédologiques, biotiques et notamment de l'action de l'homme.

Est-ce que les fluctuations climatiques finissent par aboutir à une croissance moyenne caractéristique? Quel est l'effet des variations accidentelles (ou provoquées par l'homme) de la densité sur la production totale?...

Les réponses à ces questions ne se sont dégagées que peu à peu.

Toujours est-il que cette hypothèse est aujourd'hui suffisamment admise et qu'on relie constamment les notions de station et celles de production.

C'est donc parmi les caractéristiques écologiques de la station, à travers leurs expressions, qu'on a recherché des indices de station. Les qualités d'un bon indice pouvant se résumer ainsi :

- facilité de mesure,

- bonne liaison avec la production,

- faible liaison avec d'autres variables influençant la production, ou faute de mieux, liaison avec ces autres variables assez bien connue.

\section{2. - Principaux types d'indices de productivité}

Le tableau 2 donne une vue générale des principaux types d'indices utilisés pour évaluer la productivité ou la productivité potentielle des stations. Il faut en effet distinguer la productivité réelle d'un peuplement réel constitué d'un matériel végétal précis (essence, provenance, mode d'installation et qualité de la reprise s'il s'agit de boisement) et la productivité potentielle, ou productivité optimale possible; la productivité potentielle elle-même étant soit celle d'une espèce, soit celle d'un peuplement forestier optimal, à l'exclusion d'une modification de la station trop importante avant son installation.

Il sort de mon propos de traiter de toutes les catégories d'indices. Je me bornerai à développer ici quelques aspects du problème des indices dendrométriques et des fonctions de production.

\section{3. - Indices dendrométriques et lois de croissance}

L'indice de productivité le plus utilisé est constitué dans le cas de peuplements sensiblement équiennes par la hauteur du peuplement à un âge de référence, 50 ans par exemple. Pour des raisons qui seront discutées plus loin, on choisit généralement une hauteur moyenne caractérisant les arbres dominants ou hauteur dominante. Il s'agit souvent, plus précisément, de la hauteur de l'arbre de surface terrière moyenne des 100 plus grosses tiges à l'hectare.

Le choix de cet indice, que nous prendrons comme exemple, est lié à l'existence d'un certain nombre de lois caractérisant la production en volume, c'est-à-dire à des lois de fonctionnement de l'ecosystème forestier. De plus, ce choix implique un certain nombre d'hypothèses. 
TABLEAU 2

Principaux types d'indices de productivité

\begin{tabular}{|c|c|c|c|}
\hline $\begin{array}{c}\text { Types d'indice } \\
\text { (nature des « entrées ») }\end{array}$ & Exemple & Limite d'emploi & $\begin{array}{l}\text { Principaux avantages } \\
\text { et inconvénients }\end{array}$ \\
\hline INDICES CLIMATIQUES & $\begin{array}{l}\text { - C.V.P. (Paterson) } \\
\text { - Indice de Weck }\end{array}$ & $\begin{array}{l}\text { - Essence climax } \\
\text { Très grande ré- } \\
\text { gion }\end{array}$ & $\begin{array}{l}\text { - bonne caractéristique des zones } \\
\text { de production potentielle égale, } \\
\text { - peu précis } \\
\text { - non valable pour les espèces } \\
\text { introduites } \\
\text { - ne rend pas compte des diffé- } \\
\text { rences de sols }\end{array}$ \\
\hline $\begin{array}{l}\text { INDICES « FLORISTI- } \\
\text { QUES » }\end{array}$ & $\begin{array}{l}\text { type de forêts } \\
\text { (Cajander) }\end{array}$ & $\begin{array}{l}\text { - Forêts naturelles } \\
\text { - grandes régions } \\
\text { bien zonées } \\
\text { - pour une essence } \\
\text { donnée }\end{array}$ & $\begin{array}{l}\text { - bonne corrélation avec la } \\
\text { production potentielle } \\
\text { - simplicité d'emploi } \\
\text { - critère " biologique " recou- } \\
\text { vrant donc d'autres phéno- } \\
\text { mènes que la seule production } \\
\text { (régénération par ex.) } \\
\text { - pas employable si perturbation } \\
\text { de la flore } \\
\text { - pas employable si disparition } \\
\text { de la flore (reboisement dense) } \\
\text { - problème d'étalonnage } \\
\text { - ne tient pas compte de la } \\
\text { variabilité génétique } \\
\text { - utilisable en dehors de la forêt }\end{array}$ \\
\hline $\begin{array}{l}\text { INDICES « ÉCOLOGI- } \\
\text { QUES 》 } \\
\text { - à partir des fac- } \\
\text { teurs du milieu } \\
\text { eux-mêmes } \\
\text { - à partir de plan- } \\
\text { tes indicatrices de } \\
\text { certains facteurs } \\
\text { du milieu } \\
\text { - ̀̀ partir de la no- } \\
\text { tion de groupe } \\
\text { écologique }\end{array}$ & $\begin{array}{l}\text { - liaison production } \\
\text { critères pédologi- } \\
\text { ques } \\
\text { (par exemple) } \\
\text { - notion de « conti- } \\
\text { nuum » écologi- } \\
\text { que (École améri- } \\
\text { caine) } \\
\text { - voir ci-dessous ex- } \\
\text { posé de M. } \\
\text { BECKER }\end{array}$ & $\begin{array}{l}\text { - dans une zone cli- } \\
\text { matiquement homo- } \\
\text { gène } \\
\text { - pour une essence } \\
\text { donnée } \\
\text { - sur les types de } \\
\text { station étudiée }\end{array}$ & $\begin{array}{l}\text { - corrélation assez bonne avec } \\
\text { la production potentielle } \\
\text { - pas toujours simple d'emploi } \\
\text { (dépend des facteurs consi- } \\
\text { dérés) } \\
\text { - critère biologique recouvrant } \\
\text { d'autres aspects que la seule } \\
\text { production } \\
\text { - étalonnage complexe (étude } \\
\text { longue et difficile) } \\
\text { - ne tient pas compte de la } \\
\text { variabilité génétique } \\
\text { - utilisable en dehors de la forêt }\end{array}$ \\
\hline $\begin{array}{l}\text { INDICES « DENDRO- } \\
\text { MÉTRIQUES " }\end{array}$ & $\begin{array}{l}\text { - hauteur domi- } \\
\text { nante } \\
\text { croissance cou- } \\
\text { rante en hauteur à } \\
1,30 \mathrm{~m}\end{array}$ & $\begin{array}{l}\text { - dans une zone } \\
\text { climatiquement } \\
\text { homogène } \\
\text { - pour une essence } \\
\text { donnée } \\
\text { - pour toutes les } \\
\text { stations }\end{array}$ & $\begin{array}{l}\text { - bonne corrélation } \\
\text { - ne permet pas de connaître } \\
\text { la productivité potentielle } \\
\text { - très simple d'emploi } \\
\text { - étalonnage nécessaire (tables } \\
\text { de production) } \\
\text { - tient compte de la variabilité } \\
\text { génétique } \\
\text { - non utilisable en dehors de la } \\
\text { forêt }\end{array}$ \\
\hline $\begin{array}{l}\text { INDICES MIXTES } \\
\text { Ce sont des fonctions } \\
\text { d'indices dendromé- } \\
\text { triques, de paramètres } \\
\text { de peuplement, et de } \\
\text { facteurs de milieu }\end{array}$ & $\begin{array}{l}\text { fonctions de pro- } \\
\text { duction finlan- } \\
\text { daises } \\
\text { (ex. P. Korvisto) }\end{array}$ & $\begin{array}{l}\text { - dans une très } \\
\text { large zone } \\
\text { - pour une essence } \\
\text { donnée } \\
\text { - pour l'ensemble } \\
\text { des stations de la } \\
\text { zone }\end{array}$ & $\begin{array}{l}\text { - combinent les avantages pré- } \\
\text { cédents en essayant d'éviter } \\
\text { les inconvénients de chaque } \\
\text { méthode }\end{array}$ \\
\hline
\end{tabular}


III. 31. Liaison hauteur-production totale.

C'est au forestier allemand EICHHORN (1904) et à ses successeurs qu'on doit une loi empirique très importante, connue sous le nom de loi de EıchноRN élargie * qu'on peut énoncer ainsi : la production totale en volume depuis l'origine d'un peuplement forestier est fonction de sa seule hauteur (moyenne ou mieux dominante).

Cette loi est valable pour une essence déterminée, croissant en futaie sensiblement équienne et dans le cadre d'une région climatiquement homogène, qui peut être du reste assez étendue (Wuchsgebiet des auteurs allemands).

Elle implique que la liaison entre la production totale et la hauteur est indépendante de l'âge, de la station, de la densité du peuplement.

III. 32. Indépendance vis-à-vis de l'âge et de la station. Notion de niveau de production.

A densité sensiblement égale, de très nombreuses études (MOOSMAYER, 1957; EtTER, 1949; Kramer, 1963) et d'autres, ont montré que la liaison en cause est bien indépendante de l'âge et de la station (voir fig. 2).

$$
\begin{aligned}
& \text { rod. istale } \\
& \text { rvom3/ ha }
\end{aligned}
$$

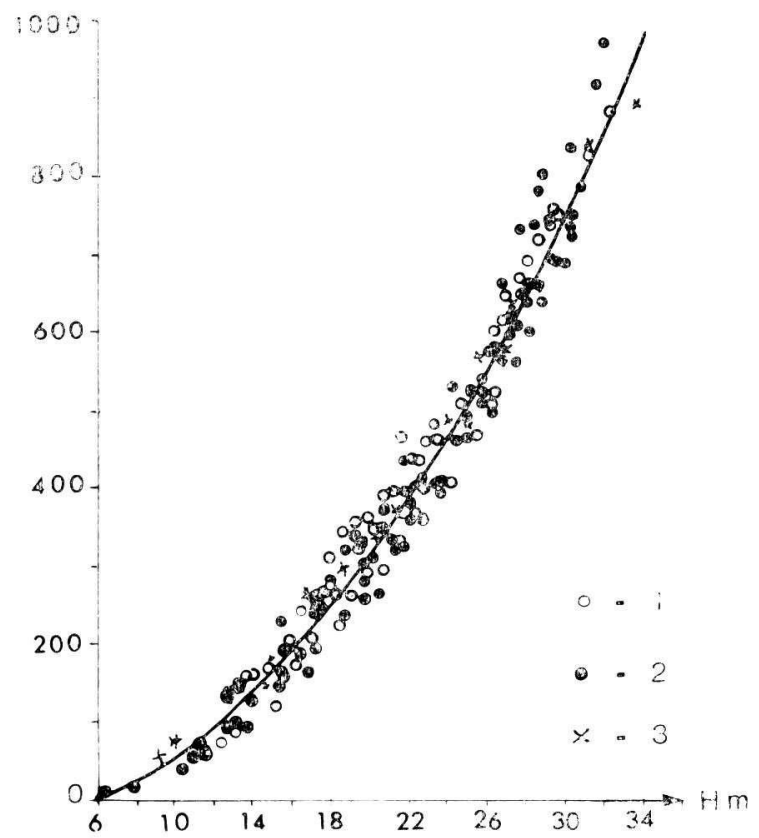

FiG. 2. - Production totale et hauteur movenne

pour le Hêtre dans le Schwabischen Alb (Moosmayer, 1957)

1 : stations calcaires (souabe du N.E.)

2 : stations sur diluvium (souabe du N.E.)

3 : stations calcaires (souabe du S.E.)

* Eichhorn avait établi sa loi pour le volume sur pied de sapinières denses. Elle a été «élargie » ensuite à d'autres essences et à la production totale. 
Ce dernier point est cependant discuté, notamment par l'École d'Assmann. Ce Professeur de l'Université de Münich a défini la notion d'Ertragsniveau (niveau de production). Il s'agit de la production totale d'un peuplement correspondant à une hauteur (moyenne ou mieux dominante) donnée.

Si la loi de EıchHorn est vérifiée, tous les peuplements considérés (même essence, même "Wuchsgebiet ») doivent avoir le même niveau de production. En fait, AssmanN affirme qu'il n'en est rien et que chaque peuplement réel possède sa propre liaison entre sa hauteur et son niveau de production. Mieux, Assmann montre à partir de placettes permanentes d'Epicéa commun en Bavière qu'à chaque Ertragsniveau correspond une station (voir fig. 3).

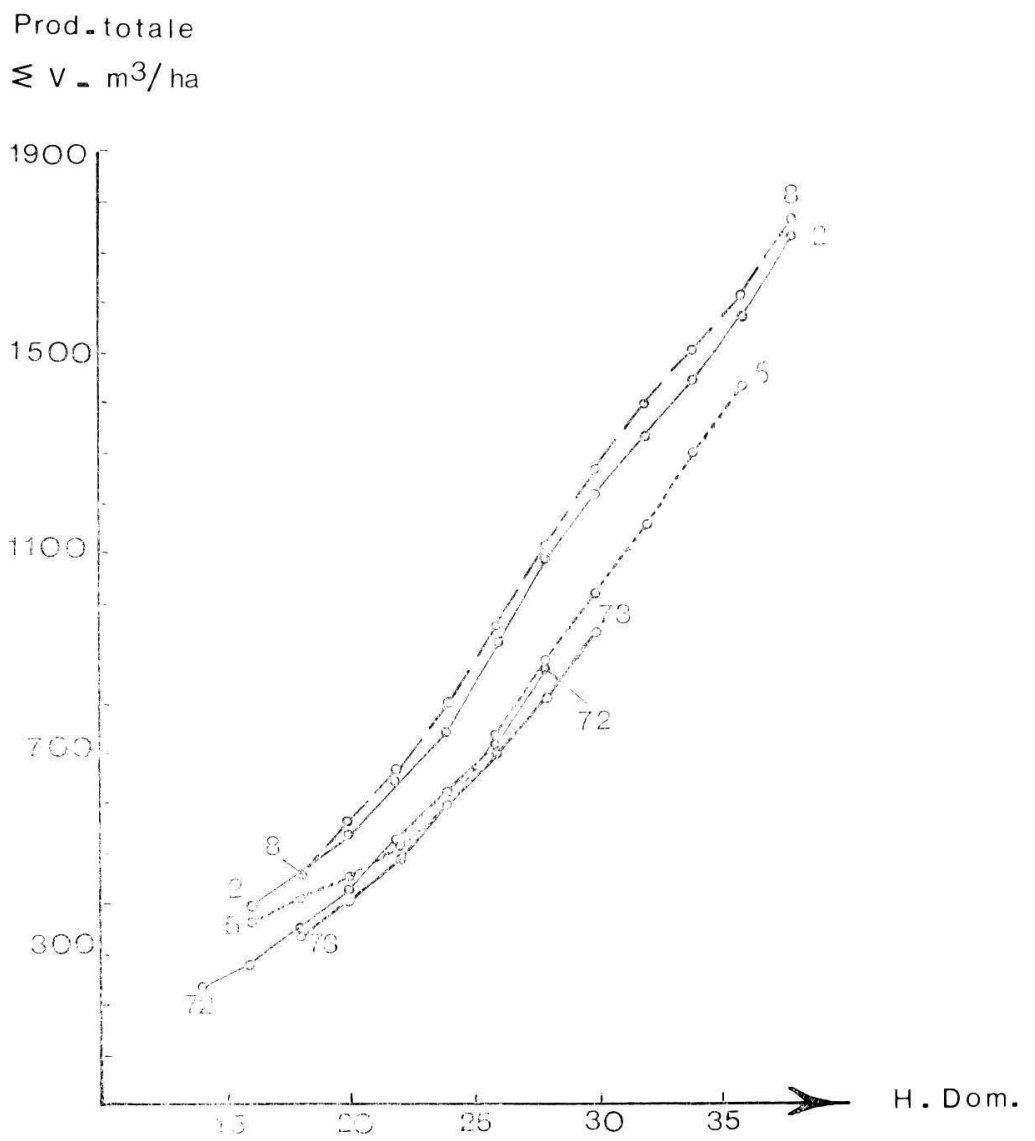

FIG. 3. - Production totale et hauteur dominante pour différentes placettes d'Épicéa Commun en Bavière (d'après Assmann - 1961)

8- 2 : terrasses bien alimentées en eau,

72-73 : plaine sur graviers, pauvre en eau,

2: profondeur des racines supérieure à $1 \mathrm{~m}$,

5 : profondeur des racines inférieure à $30 \mathrm{~cm}$. 
Poussant plus loin, il recherche l'origine de ces différences et montre que la production totale $\Sigma$ V peut-être considérée comme le produit de deux termes. D'une part, la production totale en surface terrière $\Sigma \mathbf{G}$ (somme de la surface terrière du peuplement sur pied et des surfaces terrières de tous les arbres disparus naturellement ou du fait des éclaircies); d'autre part, le produit de la hauteur par un coefficient de forme H.F.

$$
\Sigma \mathrm{V}=\text { H.F. } \Sigma \mathrm{G} \text {. }
$$

H.F. ne dépendrait que de $\mathrm{H}$, et les différences de niveau de production dépendraient en fin de compte de $\Sigma$ G. L'aptitude des peuplements à construire une surface terrière plus ou moins importante serait liée à la notion de niveau de production et notamment à la station.

Cette notion de niveau de production affine donc la loi de EıCHHORN et semble conquérir peu à peu droit de cité. Les tables de production britanniques les plus récentes en tiennent compte. Cependant, ces différences caractérisant chaque peuplement ne sont pas systématiquement attribuées à des différences de station par la plupart des auteurs (MITSCHERLich, G. 1970). Il semble bien qu'il y a en fait un ensemble de causes variées. De plus, ces différences ne portent souvent que sur de faibles écarts de production totale. La loi de EICHHORN reste donc une loi utile, approchée sans doute $( \pm 10 \%)$, mais très pratique.

\section{33. - Indépendance vis-à-vis de la densité.}

On affirme souvent que la production totale en volume ne dépend pas de l'intensité des éclaircies pratiquées, dans un très large éventail d'intensité. En fait, il va de soi que pour des densités suffisamment faibles, le potentiel productif de la station n'est plus utilisé entièrement et que la production chute. C'est encore AsSMANN et également le danois MöLLER, qui ont montré que l'accroissement courant en volume dépendait de la densité (mesurée par exemple en surface terrière). Cet accroissement passe par un maximum pour une densité donnée, c'est-à-dire pour une surface terrière optimale (voir fig. 4). La courbe pouvant être très aplatie, l'accroissement varie peu pour des densités assez voisines de l'optimum. Ces densités sont celles des sylvicultures courantes.

En toute rigueur, c'est donc la production totale correspondant à l'accroissement courant maximal qui relèverait de la loi de EichHORN. A chaque hauteur de peuplement correspondrait une densité optimale et un accroissement courant maximal. La loi fondamentale serait donc en fait cette loi d'ASSMANN-MöLler, qui correspond du reste à des considérations d'optimalité de fonctionnement de l'écosystème beaucoup mieux saisissables à ce niveau.

\section{34. - Extension de la loi.}

On peut se demander quelles sont les limites d'application de la loi de EICHHORN. Notamment, cette loi est-elle spécifique ou différente pour chaque provenance d'une même espèce? Le forestier belge NANSON (1964) a montré dans le cas d'une expérience internationale de provenances d'Épicéa commun que la loi semblait avoir un caractère spécifique. Ce caractère serait même assez large puisqu'une même loi serait valable en Allemagne de l'Ouest pour le chêne rouvre et le chêne pédonculé (KRAMER, 1963).

On peut se demander si la loi reste valable lorsqu'on modifie le potentiel productif d'une station par fertilisation par exemple. A ma connaissance, les données manquent encore pour répondre à cette question. 
Acct. Volume

$\frac{d V}{d t}$

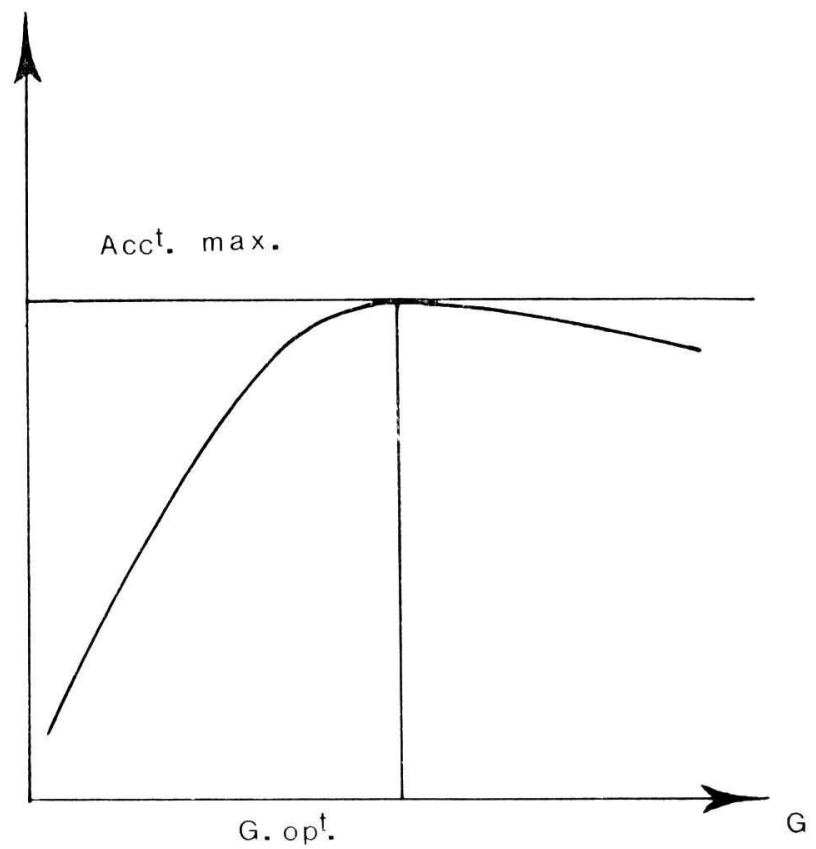

Fig. 4. - Accroissement courant en volume et densité (exprimée par la surface terrière G)

III. 35. - Autres problèmes posés par l'utilisation de cet indice.

Il faut, bien entendu, ce qui est impliqué du reste par ce qui précède, que la hauteur caractérisant le peuplement ne soit pas modifiée par la densité du peuplement. Cela explique le choix de la hauteur des plus gros arbres, pour définir une hauteur dominante, moins sujette à des variations brusques que la hauteur moyenne, lors du passage des éclaircies. Mais, il faut de plus que la croissance en hauteur soit indépendante de la densité et donc des éclaircies. Ce point est assez généralement admis, encore qu'il soit infirmé parfois, mais rarement cependant, par les résultats de telle ou telle expérience (notamment sur des pins américains [LyNCH, 1958; SMITHERs, 1961 et d'autres]).

Une autre question importante est liée au fait que les peuplements qu'on étudie ont rarement exactement l'âge de référence choisi pour définir l'indice de productivité. Il faut donc construire des faisceaux de courbes de croissance en hauteur qui correspondent à chaque indice (Site Index Curves des anglo-saxons). Passons sur les techniques utilisées, pour souligner cependant la hardiesse de l'hypothèse contenue dans cette démarche, à savoir que tous les peuplements ayant une hauteur donnée à un âge donné, ont même hauteur à tous les autres âges. Cette hypothèse est souvent infirmée par la' réalité, comme de très nombreuses études l'ont montré. 
On a donc cherché à construire des courbes de croissance en hauteur propre à une station bien définie, à un type de forêt par exemple (ILvessalo, 1927, 1937). On constate alors des différences avec les faisceaux de courbes valables pour toutes les stations, évoqués ci-dessus. Mais, on constate aussi que pour un même type de station, les courbes de différentes placettes peuvent être aussi différentes de la courbe moyenne que dans les cas où on avait rassemblé les données de plusieurs stations (JONES, J. 1969).

On accepte donc l'hypothèse ci-dessus, comme moyen, approximatif sans doute, mais pratique, de classer les peuplements.

\section{4. - Autres indices dendrométriques}

On a trouvé peu d'autres indices aussi valables que la hauteur dominante à un âge donné, et c'est généralement par comparaison avec cet indice usuel qu'on essaie de tester les autres indices possibles. C'est d'ailleurs également, soulignons-le ici, par l'intermédiaire des hauteurs dominantes que la plupart des études de liaison station-production sont effectuées, notamment au C. N. R. F. (Oswald, 1969; Decourt et Le Tacon, 1970).

Quelques essais ont été faits, notamment en choisissant comme indice ics diamètres des plus gros arbres du peuplement (DECOURT, 1967). Notons aussi l'utilisation de la croissance courante en hauteur au niveau de 1,30 m au-dessus du sol ( Five Year Growth Intercept Method » de M. W. DAY, C. F. Bey et V. J. Rudolph, 1960) qui semblent donner de très bons résultats.

\section{5. - Fonctions de croissance et indices dendrométriques}

Un des rôles importants des indices dendrométriques est de servir « d'entrée » dans la plupart des tables de production.

Les tendances les plus récentes, notamment en Finlande (Korvisto, P. 1970) visent à remplacer les tables traditionnelles par des fonctions de croissance, reliant la croissance courante à des caractéristiques du peuplement (âge, surface terrière, diamètre moyen), à l'indice traditionnel ( hauteur dominante à un âge donné) et à des caractéristiques du milicu (longueur de la saison de végétation, température, altitude, latitude, etc.).

Ces fonctions font donc intervenir des indices mixtes, à la fois écologiques et dendrométriques. A cette tendance cioivent être également rattachées les méthodes russes de classement des peuplements, d'abord par type de forêt, à l'aide de plantes indicatrices puis, dans chaque type, à l'aide des hauteurs dominantes.

Ces tendances synthétiques expliquent également que plusieurs auteurs essaient de trouver des corrélations entre indices d'essences différentes, permettant donc de prévoir la productivité d'une espèce à partir de la mesura de l'indice d'une autre. Cela souligne bien, finalement, le caractère biologique des indices de productivité dendrométriques qui mesurent la vigueur d'un composant important du tapis végétal (DOOLITTLE 1958 et autres cités par JoNFS, J. R., 1969). 


\title{
III. 6. - Indices dendronétriques et productivité primaire
}

Nul doute que de tels indices soient concevables, comme pour la productivité en volume. Les remarques faites au sujat des liaisons étroites entre la surface terrière et la biomasse laissent supposer que ces indices ne seront sans doute pas très différents de ceux utilisables pour le volume. Les études sont cependant encore trop peu nombreuses pour permettre la découverte de tels indices.

On possède cependant déjà un résumé assez complet des études de biomasse et de productivité primaire effectuées à ce jour dans le monde, grâce aux travaux de ART, H.W. et Marks, P.L. (1971). A l'aide de ces données, j'ai essayé d'effectué un classement reliant la productivité primaire à la biomasse productrice, pour différents niveaux de l'index foliaire (voir fig. 5).

N'ont été retenues dans le travail cité ci-dessus que les données comparables : biomasse des parties aériennes seules, évaluation de la productivité primaire nette par la mesure du ou des derniers cernes annuels et bien entendu mesure effective de l'index foliaire. Pour ce dernier, on a compté la surface totale des feuilles rapportée à l'unité de surface au sol, en prenant en compte toutes les faces des aiguilles ou des feuilles.

Compte tenu du caractère relativement approximatif et souvent disparate des mesures effectuées, on constate cependant, et pour des peuplements extrêmement variés (de la forêt tropicale humide aux futaies d'épicáa en paszant par les Pruniers de Pensylvanie), que l'index foliaire est en première approximation un bon indics ds productivité, à condition de le corrigar par la biomasse productrice, un pau comma dans le cas de la hauteur dans le cas de l'accroissement en volum , corrigé par la densité. II faut ajouter cependant que cet index, contrairement à la hauteur dominante notamment, est certainement sujat à des variations brusques lors du passage des coupes d'éclaircie.

Ce résultat, s`il se confirmait, soulignerait que les différences entre les stations se traduiraient plus par des possibilités différentes dentretenir un appareil foliaire important que par des différences d'efficacité de cet appareil producteur. 11 ne s'agit là, bien sûr, que d'hypothèses.

\section{SUMMAPY}

\author{
PRIMARY PRODUCTIVITY, USEFUL P.RODUCTIVITY
}

EVALUATION METHODS. PROJUCTIVITY INDEX

First $x=$ precise tha technical vocabulary. Then, wo review briefly the recent evaluation methods, neticing each time the analogy batween volune and bionas; estimation methods. We connect the concept of productivity index with the gro vth law. Finally a hypothesis is propes : : LAI might bz a good index for primary productivity. 
N. DECOURT

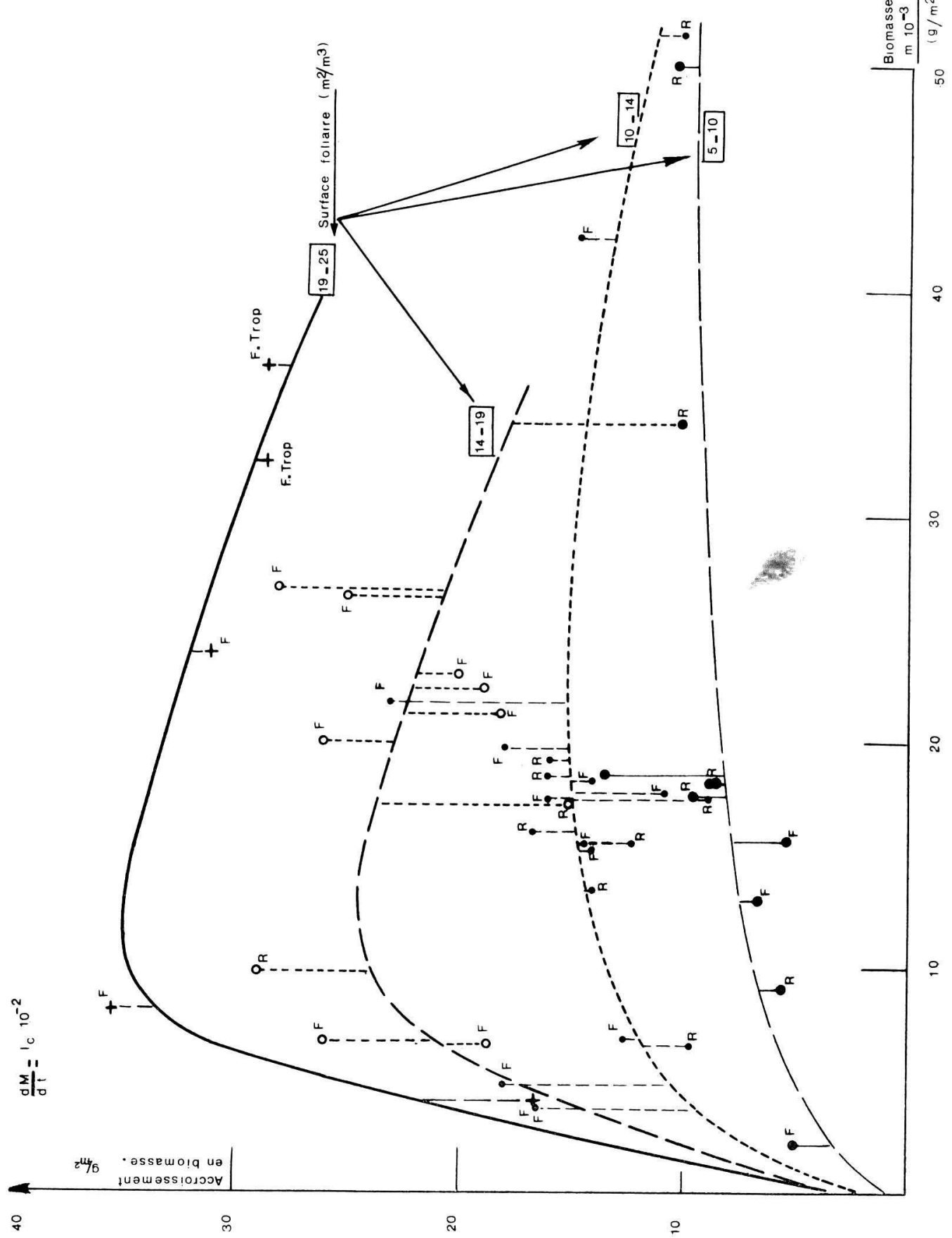

FIG. 5. - Productivité primaire nette, densité et index foliaire

F. Trop. : Forêt tropicale humide 


\section{RÉFÉRENCES BIBLIOGRAPHIQUES}

Art H. W. et Marks P. L., 1971. - A summary Table of Biomass and Net Annual Primary Production in Forest Ecosystems of the World in Forest Biomass Studies, Univ. of Maine, U.S.A.

Assmann E., 1961. - Wald Ertragskunde (B.L.V. Verlagsges., München, 1961).

Bouchon J., 1973. - Les tarifs de cubage (à paraître aux éditions de l'E.N.G.R.E.F.).

CRow T. R., 1971. - Estimation of Biomass in an Even-Aged stand, Regression and "Mean tree 》 Techniques. In Forest Biomass studies, Univ. of Maine, U.S.A.

Day M. W., Bey C. F., Rudolph V. J., 1960. - Site Index for planted Red Pine by the 5-Year Growth Intercept Method. J. Forestry, 58, 198-202.

Decourt N., 1972. - Méthode utilisée pour la construction rapide de tables de production provisoires en France. Ann. Sci. forest, 29, (1).

Decourt N., Le Tacon, 1970. - L'épicéa commun sur les plateaux calcaires de l'Est de la France. Essai de prévision de la production à l'aide de déterminations pédologiques simples. Ann. Sci. forest. vol. 27, (3).

Duvigneaud P., 1967. - La productivité primaire des écosystèmes terrestres. In Problèmes de productivité biologique, Masson.

EICHHORN F., 1904. - Beziehungen zwischen Bestandeshöhe und Bestandesmasse. A.F.J.Z., 80, 45-49.

ETtER M., 1949. - Uber die Ertragsfähigkeit verschiedener Standortstypen. Mitt. des Schweiz. Anst. für Forst. Vers. XXVI.

Huxley J., 1932. - Problems of Relative Growth. Methuen, London.

ILvesSAlo Y., 1927. - Methods for preparing yield tables. Silva Fenn. 5.

, 1937. - Growth of natural normal stands in central North-Suomi. Commun. Inst. Forest. Fenn. 24 (2).

Jones J. R., 1909. - Rewiew and Comparison of Site Evaluation Methods. U.S.D.A. Forest Service Research Paper RM-51.

KoIvisto P., 1970. - Regionality of Forest Growth in Finland. Comm. Inst. Forest. Fenn. 71-2.

Kramer H., 1963. - Der Einfluss von Grossklima und standort auf die Entwicklung von Waldbeständen... Schrif. der Forst. Univ. Gottingen.

LyNCH D. W., 1958. - Effects of stocking on site measurement and Yield of second-growth Ponderosa Pine in the Inland Empire. U.S.D.A., pap. 56, Ogden, U.T.A.M.

Madgwick H. A. I., 1971. - The Accuracy and Precision of Estimates of the Dry Matter in Stems, Branches and Foliage in an Old-field Pinus Virginiana stand. In Forest Biomass Studies, Univ. of Maine, U.S.A.

Mitscherlich G., 1970. - Wald, Wachstum und Umwelt. J. D. Sauerländer's Verlag. Frankfurt/Main.

Moosmayer U., 1957. - Zur ertragskundlichen Auswertung der Standortsgliederung im Ostteil der Schwäb. Alb. Mitt. d. Vereins f. forstl. Standortskunde, 7.

NANSON A., 1964. - Données complémentaires au sujet de l'expérience internationale sur l'origine des graines d'Épicéa en Belgique. Rech. for. Grœnendaal, Hœillaart, Travaux. Série B, $\mathrm{n}^{0} 28$.

Oswald H., 1969. - Conditions forestières et potentialité de l'Épicéa. Ann. Sci. forest., 26, (2), 1969.

Pardé J., 1961. - Dendrométrie. Éditions de l'École Nationale des Eaux et Forêts, 1961, 350 p.

RIEDACKER A., 1968. - Méthodes indirectes d'estimation de la biomasse des arbres et des peuplements forestiers. (Document C.N.R.F., 1968, distribution limitée.)

Rosen R., 1967. - Optimality Principles in Biology. (Butterworths Mathematical Texts, London 1967.)

Smithers L. A., 1961. — Lodgepole Pine in Alberta. (Can. Dep. Forest. Bull. 127, 1961.)

WECK J., 1960. - Die Waldformationsklassen der Erde und ihre potentielle substanzerzeugung. (Sond. aus Scientia 54, 6. Série 2, 1960.)

Young H. E., 1971. - Forest Biomass Studies. (Exp. Station, Univ. of Maine, Orono, U.S.A., 1971.)

\section{DISCUSSION ET INTERVENTIONS}

Après avoir mis l'accent sur les besoins de la profession forestière en ce qui concerne les tables de production et les fonctions de croissance, M. Bourgenot pose la question suivante : 
M. Bourgenot. - La courbe donnant les variations de l'accroissement en volume en fonction de la surface terrière a une caractéristique, c'est qu'elle est très aplatie au voisinage de son maximum.

On a à peu près le même accroissement entre 2 densités G 1 et G 2, assez éloignées l'une de l'autre sur la figure 4.

Cette courbe est donc très intéressante parce qu'elle montre que contrairement à ce que pensent beaucoup de forestiers, l'accroissement n'est pas une fonction indéfiniment croissante du volume sur pied. On n'a donc pas intérêt à orienter la gestion forestière vers l'cbtention de volumes dépassant un certain seuil. On a même intérêt à se tenir plutôt, du point de vue de la densité, du côté gauche de la courbe.

Mais une question se pose, que je poserai à M. Décourt. J'ai dit que nous avions, nous forestiers de terrain, un besoin urgent de tables ou fonctions de production. Or, nos peuplements réels (je cense surtout aux sapinières et pessières de montagne) présentent des densités qui sont beaucoup plus souvent surérieures qu'inférieures à la densité $\mathrm{G} \mathrm{m}$. correspondant au maximum.

Comme ce sont les peuplements réels qui servent de référence, comment faire pour obtenir des tables de production correspondant à des densités plus faibles?

M. Decourt. - Il s'agit en fin de compte da protlème ce la construction de tables de production à sylviculture variable. On avait pensé pouvoir extrapoler les données tirées des peuplements existants pour construire, à partir d'un modèle, dos tables correspondant à des éclaircies beaucoup plus fortes. 11 s.mole bien que notra nodje ne le permette pas sans danger. Il faudrait expérimenter, ca qui en natièr forestièr est très long. Une solution vers laquelie on s'orienta consistz à étuarior les rapports de conpétition entre les arbres du peuplement. Cortains se trouvent dans des conditions localas de faibiz densité, d'autres do forte densité.

Connaissant le comportam ant d'un arore en fonction do ses caractisistiques et de celles de ses voisins, y compris leurs distances, on pourra passer ensuite au pouplement, et simuler des sylvicultures variées. Cette orientation do recherche cst à la base cics études américaines de Stand model. Nous nous y engagrons également au C.N.R.F.

M. CATINOT donne ensuite quelques informations concemant les forêts iropicales :

-- Problemes de termino'ogie. La notion de production utile n'a pas le mêma sens. Le C.T.F.T. utilise pour la rechercha les notions de volume bois fort et de volune du füt. Le volume utile est le saul qui figure dans les inventaires de forêt naturalle.

- Quelques données et ordres de graindzur:

- Forêt naturelle : Volume $=300-380 \mathrm{~m}^{3} / \mathrm{Ha}$ (V. Bois Fort).

- En plantation (volume fùt),

— forêt dense - Terminalia : $300-350 \mathrm{~m}^{3} / \mathrm{ha}$,

$$
\text { — Okoumé : } 700-750 \mathrm{~m}^{3} / \mathrm{ha} \text {, }
$$

(soit $20 \mathrm{~m}^{3} /$ ha/an à 35 ans).

_ zone de savane - Eucalyptus : $120 \mathrm{~m}^{3} / \mathrm{ha}$ (en 4 ans, soit : $30 \mathrm{~m}^{3} / \mathrm{ha} / \mathrm{an}$ ).

- Quelques perspecitives de recherches. 
On a montré que beaucsup d'espèces intéressantes sur le plan forestier présentaient des cennes annuels. Ces résultats commencent à être exploités, par lecture des cernes en bout de fût, et par utilisation de tarière (type Pressler) notamment dans l'Okoumé.

M. LevéE. - Comme on l'a établi pour de nombreuses cultures herbacées, une productivité nette maximale correspond souvent à un indice de surface foliaire optimal, mais différent suivant l'architecture du feuillage. Il semble qu'il en soit de même pour les peuplements ligneux, mais on manque d'information sur ce sujet, ainsi que sur les relations entre la densité et l'indice foliaire.

M. Bouchet. - L'indice foliaire apparait bien de plus en plus comme un indice de productivité intéressant. Il serait très proîtable que bioclimatologistes et forestiers échangent leur documentation et leur expérience sur ce sujet.

- M. DeCourt : entièrement d'accord.

- M. Bonneau : Doux remarques.

1. - La fertilisation doit modificr la loi de EICHHorn, car elle modifie la forme des arbres. Par exemple dans les Landes, la fertilisation augmente la production en volume, nettement plus gue l'accroissement en hauteur.

2. - Chaque fois qu'il y a effet positif de la fertilisation, il y a augmentation de l'index foliaire. La différence de protuction entre station pourrait donc bien être due, commele dit Decourt, à une potentialité d'entretien d'une masse foliaire plus grande.

M. DECOURT. - Le fait que la production en volume augmente plus vite que l'accroissement en hauteur n'est pas contraire à la loi de EICHHORN, qui sous sa rorme allométrique implique seulement que los accroissenents relatifs de ces deux grandeurs sont porportionnels.

M. Aymonis. - Pour l'écologiste, la biomasse dans un écosystème tient compte de l'ensemble biologique présent. D'autre part, au niveau des écosystèmes, ce sont évidemment des liens dynamiques qui sont envisagés (étudiés par l'intermédiaire des bilans énergétiques). Peuton, en considérant ces faits, admettre les résultats interprétatifs de la loi d'AssMANNMöller commo significatifs au niveau du fonctionnement optimum de la phytocoenose au sein de l'écasystème considéré.

M. Decourt. - Je pense qu'effectivement une loi analogue à la loi d'Assmann-MöLter doit caractériser le niveau de production de l'écosystème, et notamment ses réactions aux influences variées qu'il subit, y compris au sein de l'écosystème lui-même. 